\title{
A NEW APPROACH TO TIME-FREQUENCY LOCALIZED SIGNAL DESIGN
}

\author{
Ahmet Kemal Özdemir, Zafer Aydın and Orhan Arıkan \\ Department of Electrical and Electronics Engineering, \\ Bilkent University, Ankara, TR-06533 TURKEY. \\ Phone : 90-312-2664307, Fax : 90-312-2664192 \\ e-mail: $\{$ kozdemir, aydinz, oarikan\}@ee.bilkent.edu.tr
}

\begin{abstract}
A novel approach is presented for the design of signals in Wigner Domain. In this method, the desired signal features in the timefrequency domain are specified in two stages. First the user specifies the spine curve around which the energy of the desired signal is distributed in the time-frequency plane. Then, the user specifies the spread of the desired signal energy around the spine. In addition to this fundamentally new way of defining the time-frequency features of the desired signal, the actual synthesis of the signal is performed in a warped fractional Fourier transform approach [1]. After obtaining the designed signal, it is transformed back to the original time domain providing the final result of the new signal synthesis technique. In contrast to the conventional algorithms, the proposed method provides very good results even if the inner cross-term structure of the desired signal is not specified.
\end{abstract}

\section{INTRODUCTION AND REVIEW OF THE EXISTING APPROACHES}

Time-frequency domain based signal design refers to synthesis of a signal from its time-frequency ( $t-f)$ distribution which is constructed by the designer to describe the desired localization of the signal energy as a joint function of time and frequency. In many fields people utilize from time-frequency domain based signal design and synthesis techniques with the purpose of filtering $[2,3]$, component extraction [4] and noise reduction [5] in the t-f plane.

Design of signals in Wigner domain received considerable interest throughout the study of time-frequency domain based signal synthesis. In the classical WD-based signal design approach the user specifies a model WD $W^{M}(t, f)$, which describes the desired distribution of signal energy in the time-frequency plane and then finds a signal $x_{W D}(t)$ whose WD resembles the model WD. Since, in general, model WD is not a valid WD, the desired signal $x_{W D}(t)$ is obtained by synthesizing a signal whose WD best fits the model WD in the least squares sense

$x_{W D}(t)=\arg \min _{\chi} \iint\left|W^{\mathcal{M}}(t, f)-W_{\chi}(t, f)\right|^{2} \mathrm{~d} t \mathrm{~d} f$,

where the WD $W_{\chi}(t, f)$ of the signal $\chi(t)$ is defined by [5]

$$
W_{\chi}(t, f)=\int \chi\left(t+t^{\prime} / 2\right) \chi^{*}\left(t-t^{\prime} / 2\right) e^{-j 2 \pi f t^{\prime}} \mathrm{d} t^{\prime} .
$$

This method provides acceptable results when the auto-term of the desired signal has a linear time-frequency support, which corresponds to no or negligible cross-term interference [5]. However if the model WD has a curved time-frequency support and the desired signal is expected to have significant cross-term interference, then the overall performance of this method is adversely affected. To illustrate this phenomenon, in Fig. 1(a), a model WD is shown to describe the auto-term structure of a desired signal. If it were possible to accurately model cross-terms, i.e., inner-interference terms [6] associated with this model WD, the designer would run the synthesis algorithm on the full WD model given in Fig. 1(b) which captures both the auto and cross-terms of the desired signal. However, because of difficulty of modeling the cross-terms, in practice the desired signal is synthesized from just the auto-term model WD given in Fig. 1(a). The WD of the synthesized signal $x_{W D}(t)$ obtained by this way is given in Fig. 1(c), where the same color encoding is used in all plots in this figure. As expected, in this example, the auto-term of the designed signal deviates from the model WD given in Fig. 1(a), especially at the middle portion where the auto-term has a large curvature.

Because of the high sensitivity of the classical WD-based signal design algorithm to inaccurate modeling of the cross-terms, a masked WD (MWD) based signal design algorithm has been developed [7]. In this approach, the fit error between the modeled and the designed WDs is minimized only at those regions of the time-frequency plane which do not contain any cross-term interference. Assuming that all the troublesome interference terms lie in the don't care region $\mathcal{D}$ of the time-frequency plane, the desired signal is synthesized by ${ }^{1}$

$$
x_{M W D}(t)=\arg \min _{\chi} \iint_{(t, f) \notin \mathcal{D}}\left|W^{\mathcal{M}}(t, f)-W_{\chi}(t, f)\right|^{2} \mathrm{~d} t \mathrm{~d} f \text { (3) }
$$

where $x_{M W D}(t)$ is the designed signal. In [7], a quasi-power algorithm is given to solve (3) iteratively. However, no convergence proof for this iterative procedure is given so far.

The MWD-based signal design algorithm usually provides better results than the classical WD-based signal design algorithm. As an illustration, the results of the classical WD and MWD-based signal design algorithms on the model of Fig. 1(a) are given in Fig. 1(c)-(d), respectively. In these plots, it is apparent that the auto-term of the designed signal by the latter algorithm provides a better fit to the model WD given in Fig. 1(a). However, the performance of the MWD-based signal design algorithm degrades if the support of the expected inner interference terms overlap with the time-frequency support of the desired signal as shown in Fig. 2(a). In this case, there is no clear choice for the don't care region. If

'When the extent of the don't care region is relatively large, an energy penalty factor is incorporated into (3) to prevent the appearance of spurious terms in the synthesized signal [7]. 
these regions are not included in $\mathcal{D}$, the performance of the MWDbased iterative design method would be close to the performance of the classical WD-based synthesis algorithm, which is not going to be satisfactory. On the other hand, if they are included in $\mathcal{D}$, this algorithm may fail to synthesize those portions of the model WD. This can be observed in Fig. 2(b), where the WD of the designed signal $x_{M W D}(t)$ is plotted after convergence of the quasi-power algorithm about in 100 iterations. Furthermore, for complicated inner interference structure, determining the extent of the don't care region can be very difficult and tedious. In addition to the difficulty in choosing the right mask, the complexity of the MWD-based synthesis algorithm is considerably higher because of its iterative computational procedure [7].

\section{A NOVEL WIGNER DISTRIBUTION BASED DESIGN METHOD USING FRACTIONAL DOMAIN WARPING}

In this paper we propose a novel and highly efficient time-frequency domain based signal design algorithm to alleviate the shortcomings of the conventional signal design methods when complicated interference terms are expected in the WD of the desired signal. The new algorithm is based on a recently developed warped fractional Fourier transformation (FrFT) algorithm [1], which can be used to map signals with complicated inner interference terms as shown in Fig. 3(a) into signals with approximately linear timefrequency supports as shown in Fig. 3(d). In the proposed method, the desired signal is synthesized in the warped FrFT domain, where its WD has considerably less interference terms. After synthesis of the signal in the transform domain, it is mapped back into timedomain providing the result of the proposed algorithm. Since in the new method, the synthesis is performed in the warped FrFT domain, there should be an easy way of characterizing the desired signal features in the transform domain instead of the original time domain. Furthermore, the mappings between the time and the warped FrFT domains should be efficient. To this purpose, the relation between the time and the transform domains should be investigated in depth in the signal design context.

\subsection{Fractional Domain Warping}

The fractional domain warping concept was first introduced in [1] to provide high resolution time-frequency representation for signals with non-linear time-frequency supports. It is the generalization of the time-domain warping to fractional domains. The warped fractional Fourier transform (FrFT) of a signal $x(t)$ is computed by replacing the time-dependence of its FrFT with a warping function $\zeta(t)$. Thus, if $x(t)$ is the time domain signal with the $a^{\text {th }}$ order FrFT $x_{a}(t), a \in \mathbb{R}$, and $\zeta(t)$ is the warping function, then the warped FrFT of the signal is given by $x_{a, \zeta}(t)=x_{a}(\zeta(t))$. The FrFT of the signal $x(t)$ used in this definition is computed by

$$
x_{a}(t) \equiv\left\{\mathcal{F}^{a} x\right\}(t) \triangleq \int K_{a}\left(t, t^{\prime}\right) x\left(t^{\prime}\right) \mathrm{d} t^{\prime},
$$

where the kernel of the transformation $K_{a}\left(t, t^{\prime}\right)$ is given in [8]. The FrFT has a number of interesting properties [8]. In this paper, we make use of its rotation property which states that, the WD of the $a^{\text {th }}$ order FrFT of a signal is the same as the WD of the original signal rotated by an angle of $a \pi / 2$ radians in the clockwise direction [8] as illustrated in Fig. 3(a)-(b) for $a=-0.8$.

In order to establish the mathematical details of the appropriate warping transformation, a spine should be defined for signals with localized time-frequency supports. In this paper, we define the spine as a curve around which the signal energy is concentrated in the time-frequency plane. As an illustration, the spines of the signals $x(t), x_{a}(t)$ with WDs shown in Fig. 3(a)-(b) are given in Fig. 4(c) and Fig. 3(c), respectively. In the context of signal design, warping transformation is most useful in fractional domains, where the spine $\psi_{a}(t)$ of the FrFT $x_{a}(t)$ is a singled valued function of time as shown in Fig. 3(c).. When the time domain support of $\psi_{a}(t)$ is $t_{1} \leq t \leq t_{N}$, the warping function and its inverse are computed by

$$
\begin{aligned}
\Gamma(t) & =\int_{t_{1}}^{t}\left[\psi_{a}\left(t^{\prime}\right)+\Delta_{f}\right] \mathrm{d} t^{\prime}, t_{1} \leq t \leq t_{N} \\
\zeta^{-1}(t) & =\Gamma(t) / f_{\psi_{a}}+t_{1}, t_{1} \leq t \leq t_{N} \\
\zeta(t) & =\Gamma^{-1}\left(f_{\psi_{a}}\left(t-t_{1}\right)\right) ; t_{1} \leq t \leq t_{N}
\end{aligned}
$$

where $f_{\psi_{a}}$ is the mean of the spine

$$
f_{\psi_{a}}=\Gamma\left(t_{N}\right) /\left(t_{N}-t_{1}\right),
$$

and $\Delta_{f}$ is the required frequency shift on the spine to ensure the invertibility of the warping operation. The amount of shift $\Delta_{f}$ is chosen such that, the frequency translated spine $\psi_{a}^{f m}(t) \triangleq \psi_{a}(t)+$ $\Delta_{f}$ is a strictly positive function of time. Thus if $\psi_{a}(t)$ is already positive, $\Delta_{f}$ is set to 0 . Note that, when a non-zero amount of shift is used in (5), instead of $x_{a}(t)$ it is more appropriate to warp its frequency modulated version $x_{a}^{f m}(t) \triangleq x_{a}(t) e^{32 \pi \Delta_{f} t}$ whose spine is $\psi_{a}^{f m}(t)$.

The effect of warping in time-frequency plane is illustrated in Fig. 3. Although the WD of $x(t)$ shown in Fig. 3(a) is cluttered with cross-terms, the WD of the warped signal $x_{a, \zeta}^{f m}(t)$ which is given in Fig. 3(d) for $a=-0.8, \Delta_{f}=2.8$ has a linear t-f support around the center frequency $f_{\psi_{a}}=2.8$ with negligible cross-term. Thus after the warping operation the inner interference terms of the signal are considerably reduced. In the next section, by utilizing this warping transformation we provide an innovative way of signal design, where the user does not have worry about the troublesome interference terms. For the sake of clarity, the steps of the proposed algorithm will be illustrated on a simulated example.

\subsection{Signal Design Using Fractional Domain Warping}

In the new signal design method, instead of directly constructing a 2-D model time-frequency distribution and synthesizing a signal based on this model, the model parameters are specified in two stages. In the first stage the designer specifies the spine curve around which the signal energy is spread in the time-frequency plane. For instance, to design a signal with an auto-Wigner term shown in Fig. 2(a), an appropriate spine would be as shown in Fig. 4(c). Note that, the user specifies a few points on the desired spine and spline interpolation [9] on the specified points provide the continuous curve for the spine. The appropriate FrFT angle $\phi$ is also shown on this figure. Then by using this FrFT angle, the spine $\psi_{a}(t)$ of the FrFT of the desired signal is computed, which is shown in Fig. 3(c) for the current design example ${ }^{2}$. In that figure, the amount of required frequency translation $\Delta_{f}$ on the rotated spine to ensure the invertibility of the warping transformation is

\footnotetext{
${ }^{2}$ For simplicity, we assume that $\psi_{a}(t)$ is a single valued function of time. Otherwise, the spine can be divided into segments which satisfy this condition. After designing signals for each spine segment, the resultant signal is obtained by combining these individual sub-signals.
} 
also shown. Then by using (5)-(8), the warping function $\zeta(t)$, its inverse $\zeta^{-1}(t)$ and the mean frequency $f_{\psi_{a}}$ are computed .

After specifying the spine and identifying an appropriate FrFT domain, the level and spread of the signal energy are specified in the warped fractional Fourier transform domain in the form of an envelope $m(t)$ and a double-sided instantaneous bandwidth $B_{i}(t)$. The user can chose $m(t)$ and $B_{i}(t)$ from a large variety of 1-D functions. In the current design example, they are chosen as shown in Fig. 4(a) and Fig. 4(b), respectively. Then, based on these 1D signal parameters, the 2-D model time-frequency distribution $W^{W \mathcal{M}}(t, f)$ is constructed in the warped domain as

$$
W^{\mathcal{W M}}(t, f)=\frac{m(t)}{\sqrt{2 \pi} \sigma(t)} \exp \left\{-\frac{\left(f-f_{\psi_{a}}\right)^{2}}{2 \sigma^{2}(t)}\right\},
$$

where $\sigma(t)=B_{i}(t) / 4$. In this construction, at each time instant the model time frequency representation is a Gaussian signal with a double-sided bandwidth $4 \sigma(t)$ as a function of frequency. Furthermore, the signal energy on the time-frequency plane around time $t$ is proportional to $m(t)$. In Fig. $4(d)$, the model timefrequency distribution constructed by using $(9)$ is given when the amplitude $m(t)$ and the instantaneous frequency $B_{i}(t)$ are chosen as shown in Fig. 4(a)-(b), respectively. Note that, there are other alternative ways of building the signal energy in the warped domain. Further research is required on this subject.

Since, in the warped domain, the constructed signal model has a linear time-frequency support, the designer can choose any of the synthesis algorithms which produces good results for this class of signals. In this paper, the WD-based signal synthesis algorithm is preferred because of its speed, robustness and good performance for this type of model time-frequency distributions with linear time-frequency supports. Thus the desired signal is synthesized in the warped FrFT domain by solving

$$
x_{a, \zeta}^{f m}(t)=\arg \min _{\chi} \iint\left|W^{M}(t, f)-W_{\chi}(t, f)\right|^{2} \mathrm{~d} t \mathrm{~d} f
$$

It is well known that, the solution of above problem is given by $x_{a, \zeta}^{f m}(t)=\sqrt{\lambda_{1}} q_{1}(t)$, where $\lambda_{1}$ is the maximal eigenvalue of

$$
D\left(t, t^{\prime}\right)=\int W^{\mathcal{W M}}\left(\left(t+t^{\prime}\right) / 2, f\right) e^{32 \pi\left(t-t^{\prime}\right) f} \mathrm{~d} f,
$$

and $q_{1}(t)$ is the corresponding eigenfunction [3]. Finally, the timedomain representation of the designed signal is computed by inversion of the warping, frequency modulation and the fractional Fourier transformation operations respectively:

$$
\begin{aligned}
x_{a}^{f m}(t) & =x_{a, \zeta}^{f m}\left(\zeta^{-1}(t)\right) \\
x_{a}(t) & =x_{a}^{f m}(t) e^{-\jmath 2 \pi \Delta_{f} t} \\
x(t) & =\left\{\mathcal{F}^{(-a)} x_{a}\right\}(t),
\end{aligned}
$$

where the FrFT order is $a=-0.8$ in the current design example and $\mathcal{F}^{a}$ is the fractional Fourier transform operator which is defined in (4). The resultant signal obtained after these operations and its WD are shown in Fig. 4(e)-(f), respectively. Note that the auto-term of the WD in Fig. 4(f) fits very closely to the model WD of the MWD-based signal design algorithm given in Fig. 2(a).

\section{CONCLUSIONS}

An efficient and versatile algorithm is proposed to design signals with localized time-frequency supports. In this new method, the synthesis is performed in a warped FrFT domain where the Wigner distribution of the desired signal has considerably less inner interference terms. On a simulation example it has been shown that, the proposed method is quite practical and easy to use. Furthermore, because there is no iterations involved, it is considerably efficient. Although it is in the class of the most efficient signal design techniques, the performance of the proposed method is superior to any of the known Wigner distribution based signal design techniques.

\section{APPENDIX}

In this appendix, steps of the proposed algorithm is given to compute samples $x\left(n T_{s}\right)$ of the desired signal. To avoid aliasing, sampling rate $1 / T_{s}$ is chosen as at least twice the Nyquist's rate.

\section{Steps of the algorithm}

1. The user specifies desired signal parameters such as the spine, rotation angle $\phi, B_{i}(t)$ and $m(t)$ as shown in Fig. 4.

2. Determine $\Delta_{f}$ then compute the warping function $\zeta(t)$, its inverse $\zeta^{-1}(t)$ and the center frequency $f_{\psi_{a}}$ by using (5)-(8).

3. Construct the model WD $W^{\mathcal{W M}}(t, f)$ by using (9).

4. Compute the entries $D[m, n]$ of the matrix $D$ as:

$c^{\mathcal{M}}[n, m]:=\int W^{\mathcal{W M}}\left(n T_{s}, f\right) e^{j 2 \pi m T_{s} f} \mathrm{~d} f$

$h[m, n]:=\operatorname{sinc}(m-1 / 2) \operatorname{sinc}(n-1 / 2)$

$D[m, n]:=\sum_{\left(m^{\prime}, n\right)^{\prime}} h\left[m-m^{\prime}, n-n^{\prime}\right] c^{\mathcal{M}}\left[m^{\prime}+n^{\prime}+1^{\prime}, m^{\prime}-n^{\prime}\right]$ $+c^{\mathcal{M}}[m+n, m-n]$

5. Obtain the maximum eigenvalue $\lambda_{1}$ of the matrix $D+D^{H}$ and the corresponding eigenvalue $q_{1}$. Then the even and the odd indexed samples of $x_{a, \zeta}^{f m}\left(n T_{s}\right)$ are obtained by:

$x_{a, \zeta}^{f m}\left((2 n) T_{s}\right)=\sqrt{\lambda_{1}} q_{1}[n] / 2$

$x_{a, \zeta}^{f m}\left((2 n+1) T_{s}\right)=\sum_{n^{\prime}} \operatorname{sinc}\left(n-n^{\prime}+1 / 2\right) x_{a, \zeta}\left(2 n^{\prime} T_{s}\right)$

6. Interpolate samples $x_{a, \zeta}^{f m}\left(n T_{s}\right)$ to obtain

$x_{a}^{f m}\left(n T_{s}\right):=x_{a, \zeta}^{f m}\left(\zeta^{-1}\left(n T_{s}\right)\right)$

7. Demodulate samples $x_{a}^{f m}\left(n T_{s}\right)$ to obtain $x_{a}\left(n T_{s}\right):=x_{a}^{f m}\left(n T_{s}\right) e^{-j 2 \pi \Delta_{f} n T_{a}}$

8. Compute samples of $x(t)$ by using fast FrFT algorithm [10]: $x\left(n T_{s}\right):=\left\{\mathcal{F}^{(-a)} x\right\}\left(n T_{s}\right)$

\section{REFERENCES}

[1] A. Kemal Özdemir, L. Durak, and O. Ankan, "High resolution time-frequency analysis based on fractional domain warping," Proc. IEEE Int. Conf. Acoust. Speech Signal Process., vol. 6, pp. 3553-3556, May 2001.

[2] F. Hlawatsch ed W. F. G. Mecklenbrauker, The Wigner distribution-theory and applications in signal processing, Elsevier Science Publishers, 1997.

[3] G. F. Boudreaux-Bartels and T. W. Parks, "Time-varying filtering and signal estimation using Wigner distribution synthesis techniques," IEEE Trans. Acoust., Speech, and Signal Process., vol. ASSP-34, no. 3, pp. 442-451, June 1986.

[4] W. Krattenhaler and F. Hlawatsch, "Time-frequency design and processing of signals via smoothed wigner distributions," IEEE Trans. Signal Process., vol. 41, no. 1, pp. 278-287, May 1993.

[5] L. Cohen, Time-frequency analysis, Prentice Hall, 1995.

[6] F. Hlawatsch and G. F. Boudreaux-Bartels, "Linear and quadratic time-frequency signal representations," IEEE Signal Processing Magazine, vol. 9, no. 4, pp. 21-67, Apr. 1992. 
[7] F. Hlawatsch, A. H. Costa, and W. Krattenhaler, "Timefrequency signal synthesis with time-frequency extrapolation and don't-care regions,". IEEE Trans. Signal Process., vol. 42, no. 9, pp. 2513-2520, Sept. 1994.

[8] Haldun M. Ozaktas, Zeev Zalevsky, and M. Alper Kutay, The Fractional Fourier Transform with Applications in Optics and Signal Processing, John Wiley \& Sons, 2001.

[9] M. Unser, "Splines a perfect fit for signal and image processing," IEEE Signal Processing Magazine, vol. 16, no. 6, pp. 22-38, Nov. 1999.

[10] H. M. Ozaktas, O. Ankan, M. A. Kutay, and G. Bozdagi, "Digital computation of the fractional Fourier transform," IEEE Trans. Signal Process., vol. 44, no. 9, pp. 2141-2150, Sept. 1996. (a)

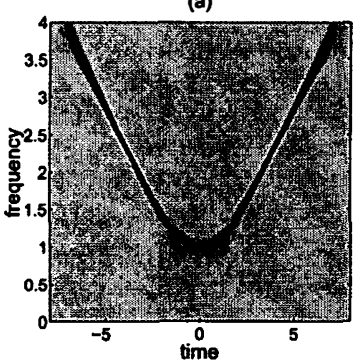

(c)

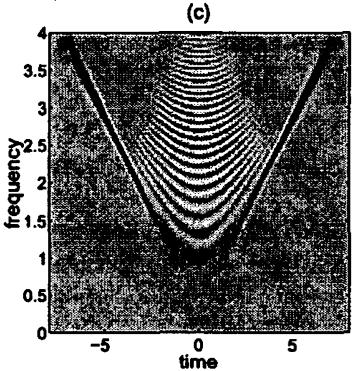

(b)

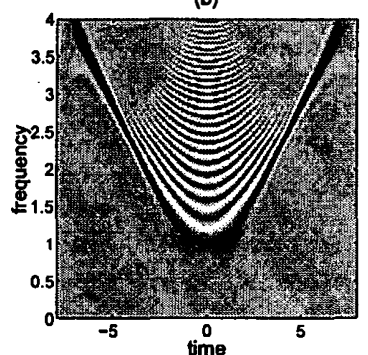

(d)

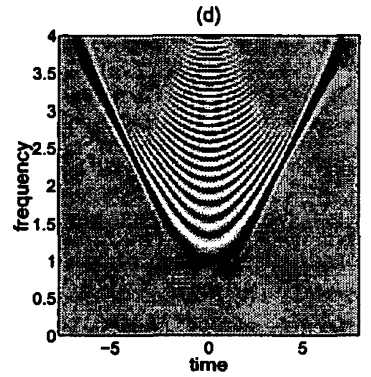

Fig. 1. (a) A model WD and (b) the corresponding full WD model including inner-interference terms, (c)-(d) The WDs of the synthesized signals by using the WD and MWD-based signal design algorithms on the model WD given in (a), respectively.
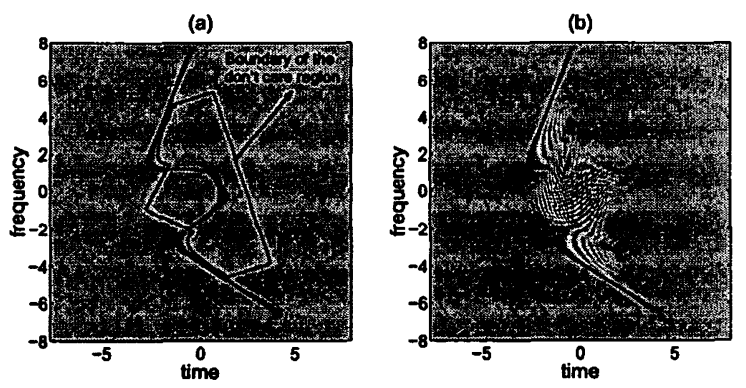

Fig. 2. (a) A model WD and support of expected interferenceterms in the desired signal, (b) the result of the MWD-based signal design algorithm on the model WD given in (a).

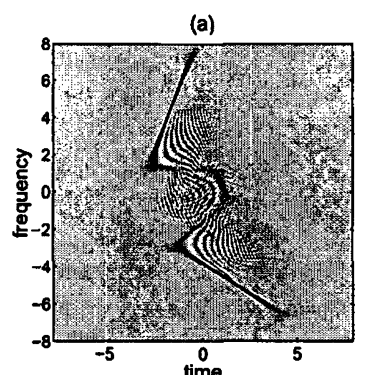

(c)
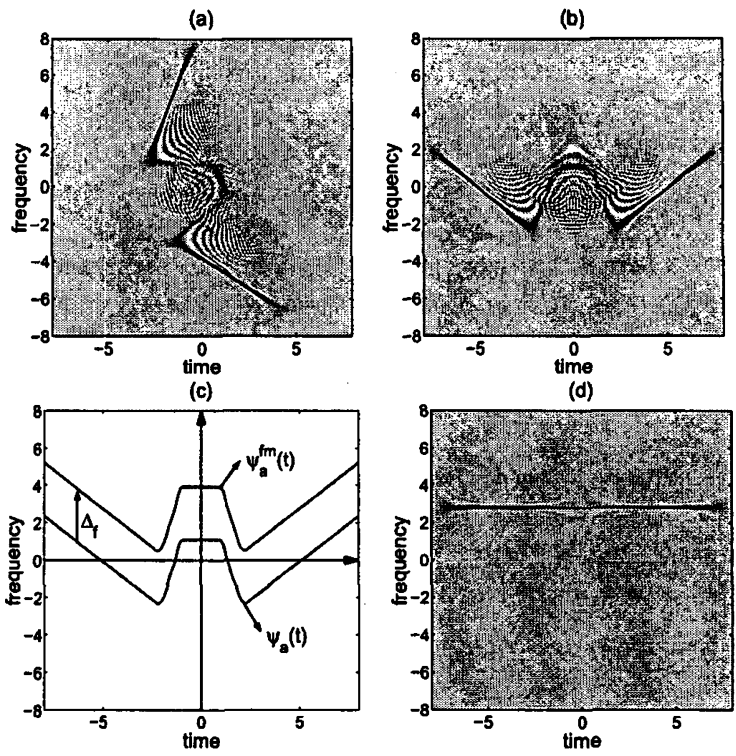

(d)

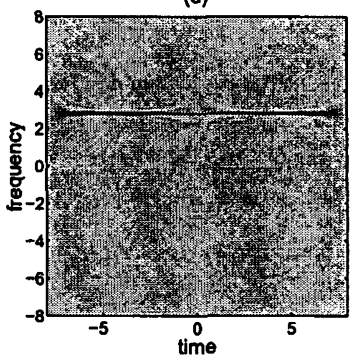

Fig. 3. (a) The WD of the signal $x(t)$, (b) the WD of the $a=$ $(-0.8)^{\text {th }}$ order FrFT $x_{a}(t)$ of $x(t)$, (c) the spines $\psi_{a}(t), \psi_{a}^{f m}(t)$ of the signals $x_{a}(t)$ and $x_{a}^{f m}(t)=x_{a}(t) e^{j 2 \pi \Delta_{f} t}$ for $\Delta_{f}=2.8$, (d) the WD of the warped signal $x_{a, \zeta}^{f m}(t)=x_{a}^{f m}(\zeta(t))$.
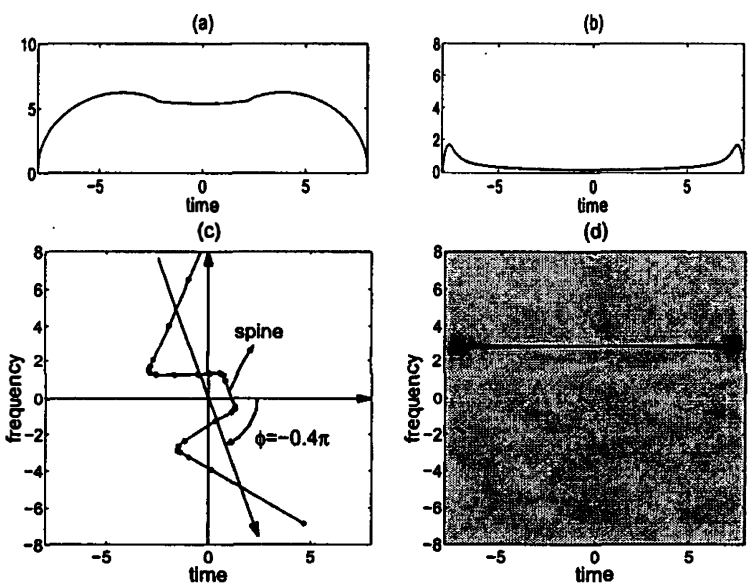

(d)

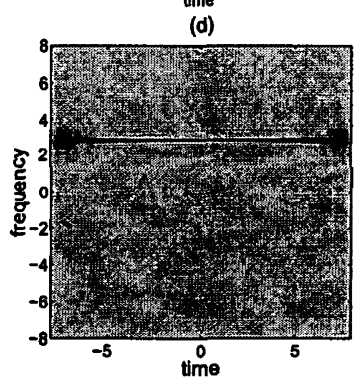

(e)
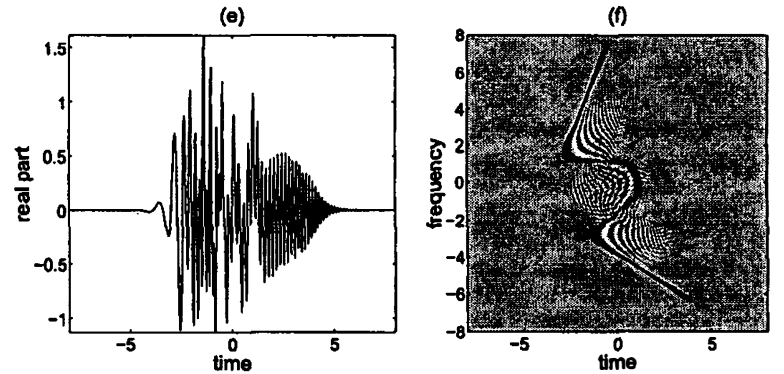

Fig. 4. The user specified (a) amplitude $m(t)$, (b) instantaneous bandwidth $B_{i}(t)$ and (c) signal spine; (d) the corresponding model WD $W^{\mathcal{W M}}(t, f)$ in the warped FrFT domain, (e) the time-domain representation of the synthesized signal $x(t)$ based on the model WD given in (d) and (f) its WD $W_{x}(t, f)$. 